\title{
EDIM-TKTL1 blood test: a noninvasive method to detect upregulated glucose metabolism in patients with malignancies
}

\author{
Oliver Feyen*1‡, Johannes F Coy ${ }^{\ddagger}$, Vikas Prasad ${ }^{2,3 \ddagger}$, Ralf Schierl', Joerg Saenger ${ }^{4}$ \\ \& Richard P Baum² \\ ITAVARLIN AG, Landwehrstrasse 54, D-64293 Darmstadt, Germany \\ 2Department of Molecular Radiotherapy, Center for Molecular Imaging, Zentralklinik Bad Berka, \\ Robert-Koch-Allee 9, D-99437 Bad Berka, Germany \\ ${ }^{3}$ Department of Nuclear Medicine, Charite University Hospital, Augustenburgerplatz 1, 13353 Berlin, Germany \\ 4Institute of Pathology, Robert-Koch-Allee 9, D-99437 Bad Berka, Germany \\ ¥Authors contributed equally \\ *Author for correspondence: Tel.: +4961519505550 n. Fax: +4961519505551 = o.feyen@tavarlin.de
}

Aim: To determine whether the TKTL1 protein epitope detection in monocytes (EDIM) test allows detection of upregulated glucose metabolism in malignancies. Materials \& methods: The EDIM-TKTL1 blood test was conducted in 240 patients with 17 different confirmed or suspected malignancies. Test scores were compared with ${ }^{18} \mathrm{~F}$-fluoro-2-deoxy-D-glucose (FDG)-PET/computed tomography (CT) results. Results: EDIM-TKTL1 score and FDG-PET results showed a concordance of $90 \%$ with a sensitivity of $94 \%$ and specificity of $81 \%$. Including CT data, all values were enhanced. A subgroup analysis of non-small-cell lung cancer patients showed a significant correlation between the EDIM-TKTL1 score and the primary tumor size determined by FDG-PET/CT. Conclusion: EDIM-TKTL1 blood test revealed good concordance with FDG-PET/CT results in patients with malignancies demonstrating its efficacy to detect upregulation of glucose metabolism in primary tumors or metastases.

Upregulated glucose metabolism in tumors and metastases can be visualized by PET using ${ }^{18}$ F-fluoro-2-deoxy-D-glucose (FDG) [1]. This in vivo technique is a powerful imaging tool available for clinical use, especially in oncology. The rationale for using FDG-PET in oncology is based on the observation that malignant cancer cells show increased glucose uptake and upregulation of proteins and enzymes for glucose metabolism compared with normal tissues [2-6]. As this increased glucose metabolism is an important event in the process of malignant transformation and often precedes morphological tissue changes, FDG-PET is of value for diagnosis and in vivo visualization of cancer. FDG-PET in combination with computed tomography (FDG-PET/CT) allows for precise localization and identification of malignancies and is increasingly and efficiently used for patient management in oncology, for example, in lung cancer, breast tumors, lymphoma and others [7-11]. Based on detailed reviews among meta-analyses of pertinent clinical data, recommendations on the use of FDG-PET scans have been worked out for various tumor entities and disease stages [12,13].

The cause of increased FDG-uptake in tumors has been extensively studied and is found to be related to coordinated upregulation of different metabolic pathways including core glycolysis, pentose phosphate and carbon fixation pathways [14]. An activated pentose phosphate pathway results in altered tumor metabolism, malignant transformation and increased tumor proliferation and progression. It is associated with enhanced angiogenesis, tumor migration and metastasis [15]. Transketolases are key enzymes in the pentose phosphate pathway and it has already been shown that the Transketolase-like-1 (TKTL1) gene is strongly upregulated at the mRNA and protein levels in cancer cells and tumors/metastases [16-22].

TKTL1 contributes to a malignant phenotype through increased glucose metabolism even in the presence of oxygen (Warburg effect/aerobic glycolysis) and HIF1 $\alpha$ accumulation [23]. For rapid cell growth and full viability of human tumor cells, TKTL1 is required [24]. Inhibition of TKTL1 gene expression results in inhibition of tumor cell growth and proliferation as well as reduced glucose metabolism [23-25].

TKTL1 gene overexpression in cancer cells has been correlated with a more malignant cancer phenotype [17], increased rate of metastasis $[18,19,21,22]$ and poor prognosis in patients with

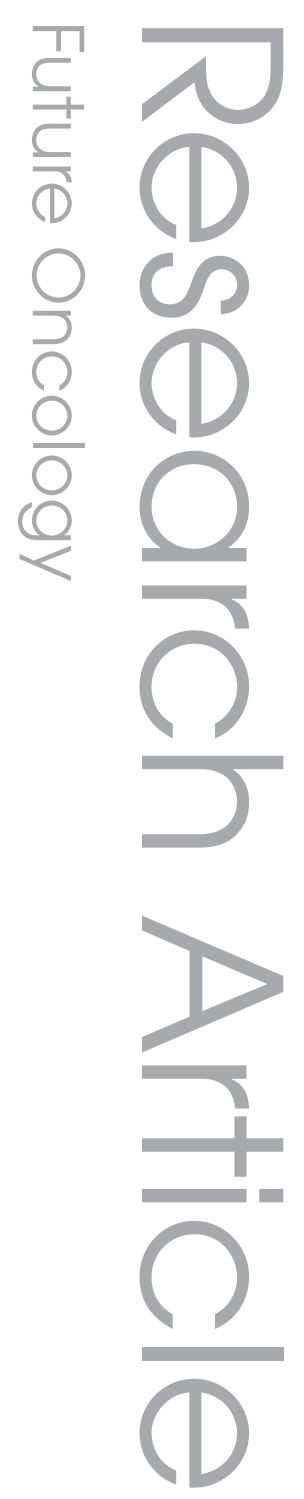

\section{Keywords}

- blood test - diagnostic

- EDIM - FDG-PET - glucose

metabolism $=$ malignancy

- oncology - TKTL1

- transketolase-like-1

- upregulation

\section{Future : Medicine}


lung, colon, rectal, urothelial, ovarian and ocular adnexal tumors $[16,19,21,22,26,27]$. For example, in patients with locally advanced rectal cancer treated with intensified neoadjuvant chemotherapy, radiotherapy and targeted therapies, high TKTL1 expression correlated with poor prognosis, the development of metastases or local recurrence [21]. In patients with colorectal cancer, TKTL1 expression is higher in more advanced cancers and overexpression of TKTL1 correlates with lymph node involvement and with poor prognosis $[16,28]$. All in all, TKTL1 can be used as a biomarker that indicates activation of pentose phosphate pathway and increased glucose metabolism in malignancies.

The aim of this study was to evaluate the diagnostic efficacy of a new blood test for the detection of TKTL1 in monocytes or macrophages in patients with malignancies. This new test is based on the published epitope detection in monocytes (EDIM) technology [29-31], which utilizes the fact that activated macrophages phagocytize and present tumor-related material. Those activated macrophages, which contain intracellular tumor epitopes, can be detected by specific antibodies using flow cytometry. The EDIM-TKTL1 blood test allows detection of circulating activated macrophages in the peripheral blood and determination of the proportion of those macrophages that present intracellularly located TKTL1 antigen. Results are compared with FDG-PET and FDG-PET/CT findings, which is the current gold standard for the in vivo determination of glucose metabolism in malignancies.

\section{Materials \& methods Patients}

Overall, in this prospective study, 240 consecutive individuals scheduled for FDG-PET/CT with confirmed or suspected tumors/metastases were included. Furthermore, 122 blood samples from 117 blood donors (blood donation service, Darmstadt, Germany) were tested to determine the distribution of TKTL1 scores in the normal population. Analytical performance for interand intraspecific reliability of the EDIM-TKTL1 blood test was undertaken with an additional 62 individuals.

\section{Consent \& ethics}

FDG-PET/CT imaging was performed as part of the routine clinical investigation independent of the TKTL1 study. Blood samples from patients and healthy volunteers were taken only after written, informed and signed consent had been obtained. The study was performed after approval by the institutional review board of the hospital and in accordance with the ethical guidelines laid out in the Declaration of Helsinki.

\section{Blood samples}

Blood samples $(3 \mathrm{ml})$ were collected in EDTA tubes, anonymized and then processed within $24 \mathrm{~h}$, blinded to the clinical data.

\section{Immunocytochemical staining}

Monoclonal antibodies were added to the blood sample to label the monocytes/macrophages: allophycocyanin-conjugated antibody against CD14 (clone M $\varphi$ P9, BD Biosciences, Heidelberg, Germany) and a fluorescein isothiocyanate-conjugated antibody against CD16 (clone NPK15, BD Biosciences). After a first staining step, cells were fixed (IntraPrep, Beckman Coulter, Krefeld, Germany) and washed once (CellWash, $\mathrm{BD}$ Biosciences). After resuspendation, permeabilization reagent (IntraPrep, Beckman Coulter) was added and incubated in the dark. To label intracellular TKTL1, cells were incubated subsequently with a phycoerythrin (PE)conjugated TKTL1 antibody (clone JFC12T10, TAVARLIN, Germany). As a negative control, an aliquot of the cell suspension was incubated with PE-labeled mouse immunoglobulins (BD Biosciences) to reveal background staining by the conjugated primary antibody. To control the specificity of the TKTL1 antibody, a staining to identify another intracellular epitope (Apo10) was performed using monoclonal Apo10 antibody (clone DJ28D4, TAVARTIS GmbH, Otzberg, Germany) rather than the TKTL1 antibody.

\section{Flow cytometry measurements}

Samples were analyzed by fluorescence-activated cell sorting using three fluorescence-activated cell sorting devices (CantoII, BD Biosciences; Calibur, BD Biosciences; FC500, Beckman Coulter). To select 10,000 monocytes from the whole blood sample, a gate was drawn in the forward scatter/side scatter dotplot due to its size (forward scatter) and granularity (side scatter) to exclude lymphocytes next to debris and apoptotic cells. The monocytes $\left(\mathrm{CD} 14^{+}\right)$and activated macrophages $\left(\mathrm{CD} 14^{+} / \mathrm{CD} 16^{+}\right)$were first identified using the presence of CD14 and side scatter to exclude granulocytes showing negative staining for CD14. Next, the selected monocyte population was displayed for the expression of CD14 and CD16. In new dotplots, a stretched (low to high intensity) $\mathrm{CD} 14^{+}$population could 
be observed, representing the monocytes of this sample. The second population shows stretched (low to medium) CD14 labeling intensity, with a medium to high CD16 signal. This $\mathrm{CD} 14^{+} / \mathrm{CD}_{16}{ }^{+}$population represents the mature, circulating macrophages $[30,31]$.

After gating, the percentage of TKTL1 ${ }^{+}$ macrophages $\left(\mathrm{CD} 14^{+} / \mathrm{CD} 6^{+} / \mathrm{TKTL1}^{+}\right)$was detected and then used as a basis to determine the TKTL1 score. Fluorescein isothiocyanate, $\mathrm{PE}$ and allophycocyanin signals were recorded as logarithmically amplified data. Analysis was performed using FACSDiva software v6.1. (BD Biosciences).

\section{FDG-PET/CT}

\section{Patient preparation}

All patients were fasted for at least $6 \mathrm{~h}$ prior to the injection of FDG and were prepared under strict guidelines as published by the European Association of Nuclear Medicine [32].

\section{Imaging \& image analysis}

${ }^{18} \mathrm{~F}_{-} \mathrm{FDG}_{\text {scint }}$ was made available from the radiopharmacy of the Zentralklinik, Bad Berka. On average, each patient received 4.5-5 MBq of ${ }^{18} \mathrm{~F}-\mathrm{FDG}$ per kilogram of body weight. Images were acquired approximately $60 \mathrm{~min}$ postinjection. Depending upon the weight of the patient, FDG-PET images were acquired from 2-3 min per bed position. All patients were examined on a dual-modality PET/CT tomography (Biograph Duo, Siemens Medical Solutions, Erlangen, Germany). The CT component consists of a two-row spiral CT system with a maximum continuous scan time of $100 \mathrm{~s}$ and a maximum rotation speed of $75 \mathrm{rpm}$. The PET component is based on a full-ring luthetium orthosilicate PET system. Details of the PET/CT imaging procedure, data reconstruction and image processing have already been described elsewhere [33].

In short, the maximum intensity projection images were visually inspected in varying scales. Thereafter, each single transversal slice was viewed cranio-caudally in combination with the corresponding CT image; the fused image slice and each focal, abnormal tracer uptake was recorded by slice number and anatomic localization. Finally, a manually selected region of interest was automatically drawn on a single slice of the reconstructed PET images using a 50\% standardized uptake value (SUV) threshold and the provided software (Siemens esoft). For semiquantitative analysis, maximum and mean SUV $\left(S U V_{\text {max }}\right.$ and $S U V_{\text {mean }}$, respectively), molecular tumor volume and molecular tumor index were calculated. FDG-PET studies were read and interpreted with full knowledge of all clinical data by two experienced nuclear medicine physicians with 18 years (RP Baum) and 5 years (V Prasad) of PET experience, respectively. The FDG-PET findings were confirmed either by histopathology within 2 weeks of imaging (preoperative staging) or by clinical follow-up at least 6 months later.

\section{Statistical analysis}

Specificity, sensitivity, positive predictive value (PPV), negative predictive value (NPV) and concordance was performed using Microsoft Excel (Microsoft, WA, USA) in order to determine the accordance between the EDIM-TKTL1 score and FDG-PET and FDG-PET/CT data, respectively. As a quantitative parameter, the $\mathrm{SUV}_{\max }$ and the metabolic tumor volume were correlated. For drawing the scatter plots and the receiver operating curve, SPSS version 13 was used. Analysis of intraserial and interdevice precision was performed with two-way model II analysis of variance with random effects for unbalanced data. Correlation between primary tumor stage and the EDIM-TKTL1 score was tested using the Kruskal-Wallis test.

\section{Results}

\section{Patients}

This study included 240 consecutive participants (129 men and 111 women) with an average age of 62 years (19-85 years). The following different tumor entities were evaluated: non-small-cell lung cancer (NSCLC; $\mathrm{n}=139$ ), small-cell lung cancer (SCLC; $n=4)$, breast $(n=41)$, colorectal $(n=18)$, carcinoma of unknown primary $(n=9)$, lymphoma $(n=4)$, cholangiocarcinoma $(n=3)$, esophageal $(n=3)$, stomach $(n=2)$, salivary glands $(\mathrm{n}=2)$ and thyroid $(\mathrm{n}=2)$ cancers and one each of melanoma, renal, palatine tonsil, hepatocellular, germ cell tumor and prostate cancer. Seven patients (six of them with lung disease) had histologically and clinically documented nonmalignant diseases including granulomatous lung disease, benign lung nodule, benign pleural effusion, sarcoidosis, asbestosis, hamartoma and osteochondrosis.

\section{Intracellular TKTL1 staining}

To evaluate the specificity of the TKTL1 antibody, first PE-conjugated isotype of TKTL1 (murine IgG) staining was performed to determine background signal intensity (Figure 1A). The amount of TKTL1-containing activated 

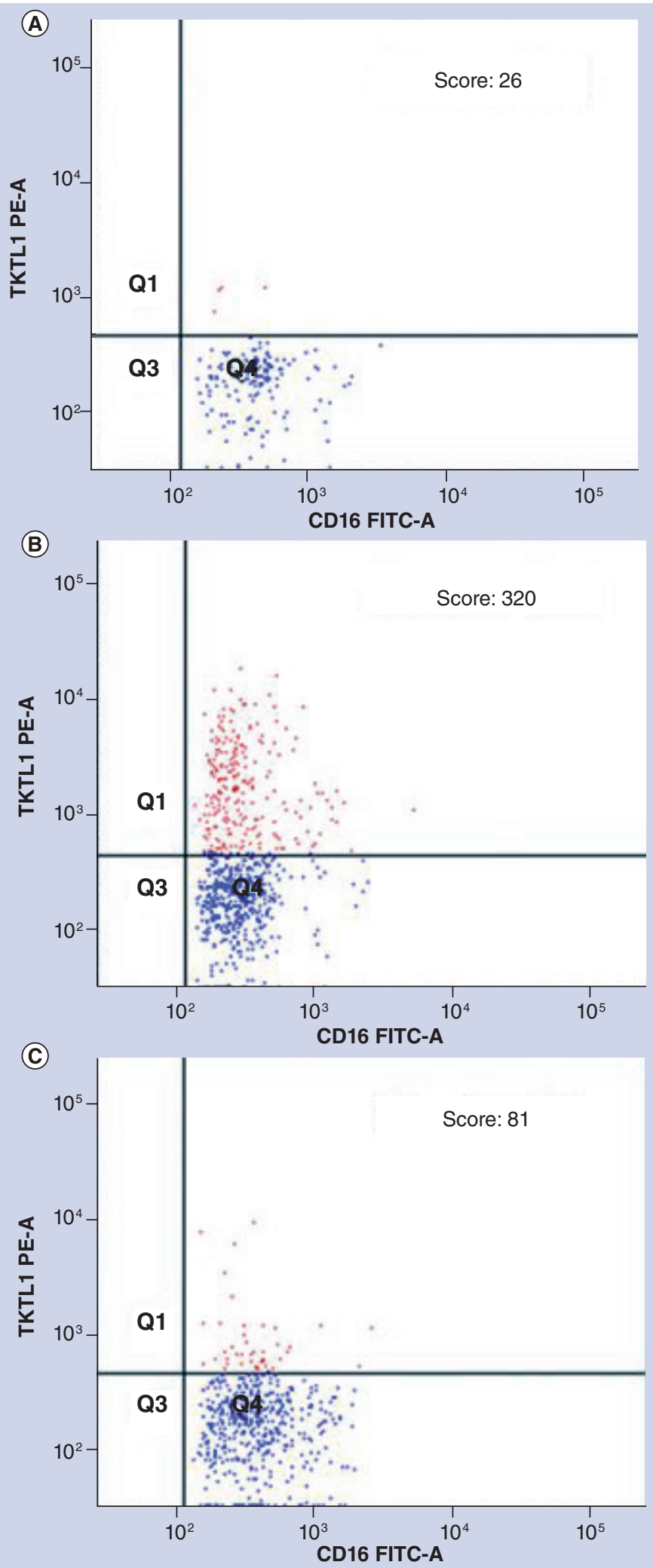

Figure 1. Dotplots of TKTL1/isotype (phycoerythrin axis, red population) and CD16 (FITC axis, blue population) staining. (A) Isotype control (background staining). (B) Patient with malignant tumor. (C) Patient with benign tumor.

FITC-A: Fluorescein isothiocyanate area; PE-A: Phycoerythrin area. macrophages was then determined by intracellular staining of the $\mathrm{CD} 14^{+} / \mathrm{CD} 16^{+}$monocyte population. Specific TKTL1-containing macrophages were identified as $\mathrm{CD} 14^{+} / \mathrm{CD} 16^{+} /$ $\mathrm{TKTL}^{+}$cells. The arithmetical mean of the absolute number of these triple-positive macrophages was 159 (range: 19-894). Finally, the relative amount of this population in relation to all macrophages served as the basis for calculating the TKTL1 score. An example of dotplots of patients with malignant tumors is shown in Figure 1B. A significant cellular fraction is found with an increased signal, indicative of intracellular TKTL1 staining. In samples from patients with benign tumors, little or no signal is observed over background intensity (FIGURE 1C).

\section{Determination of EDIM-TKTL1 cut-off score by comparison of EDIM-TKTL1 score \& FDG-PET results}

The receiver operator curve (Figure 2) was performed including all 240 individuals (with confirmed malignancies and nonmalignant truenegative cases as standard) compared with the EDIM-TKTL1 blood test. The analyses showed that the best cut-off score for EDIM-TKTL1 test was 119 , resulting in a sensitivity of $94 \%$ and specificity of $81 \%$; the area under the curve was found to be 0.89 . As this test is primarily being developed as a screening method, higher sensitivity with acceptable specificity was the primary reason for fixing the cut-off at 119 . EDIM-TKTL1 scores below 119 were defined as TKTL1 'negative', those equal and above 119 were defined as 'positive'.

\section{Validation of the EDIM-TKTLI blood test}

The cut-off score of 119 was then applied to the analysis of 122 blood samples of 117 potentially healthy people (67 men and 50 women) with an average of 35 years (18-67 years). Blood samples included five duplicates with an interval of 4-8 weeks. Of the blood samples, 94.3\% had a negative EDIM-TKTL1 score. Therefore, $5.7 \%$ of blood donors showed a positive EDIM-TKTL1 score, that is, a positive result in the EDIM-TKTL1 blood test confirming the expected 95:5 distribution of negative to positive cases.

Repeatability of the EDIM-TKTL1 blood test was evaluated by measurement of several replications, both by the authors and in cooperation with three external laboratories (Krefeld and Heidelberg, Germany, and Rapperswil-Jona, Switzerland). Intraserial and interdevice precision were analyzed from 197 measurements in 
62 samples in one to six replicates on three flow cytometry devices (CantoII, BD Biosciences; Calibur, BD Biosciences; FC500, Beckman Coulter). Statistical analysis resulted in an intraserial coefficient of variation, interdevice and total coefficient of variation of 7.7, 4.9 and $9.1 \%$, respectively. Overall, only $1 \%$ (2 out of 197 ) resulted in an opposite (negative) score range (TABLE $\mathbf{1})$.

\section{Comparison of EDIM-TKTL1 scores with FDG-PET results as standard (with \& without benign diseases)}

Comparison of the 240 patients either with confirmed or suspected tumors/metastases as well as nonmalignant diseases (alternatively without these seven benign tumors; $\mathrm{n}=233$ ) omitting the CT data (Figure 3 \& Table 2 ) showed that 150 out of 240 (150 out of 233) patients were true-positive, that is, both EDIM-TKTL1 blood test and FDG-PET imaging showed positive results. Of these patients, 65 out of 240 (58 out of 233) were true-negative, in both cohorts 15 patients were false-positive (FP) in EDIM-TKTL1, whereas ten patients were false-negative. There was a significant concordance between the EDIM-TKTL1 score and FDG-PET results, since 215 out of 240 $(90 \%$ [ 208 out of $233=89 \%$ ]) of the patients had identical positive or negative results in their respective EDIM-TKTL1 blood test and FDG-PET examination. Overall, the diagnostic performance revealed a sensitivity of $94 \%$ (regarding 'malignant patients', i.e., without benign tumors: $94 \%$ ) and specificity of $81 \%$ (80\%). The PPV was $91 \%$ (91\%), while the NPV was found to be $87 \%$ ( $85 \%$; TabLe 2$)$.

\section{Comparison of EDIM-TKTL1 score \& FDG-PET including $\mathrm{CT}$ results}

Data of those 15 patients positive in EDIM-TKTL1 but negative in FDG-PET (i.e., FP patients)

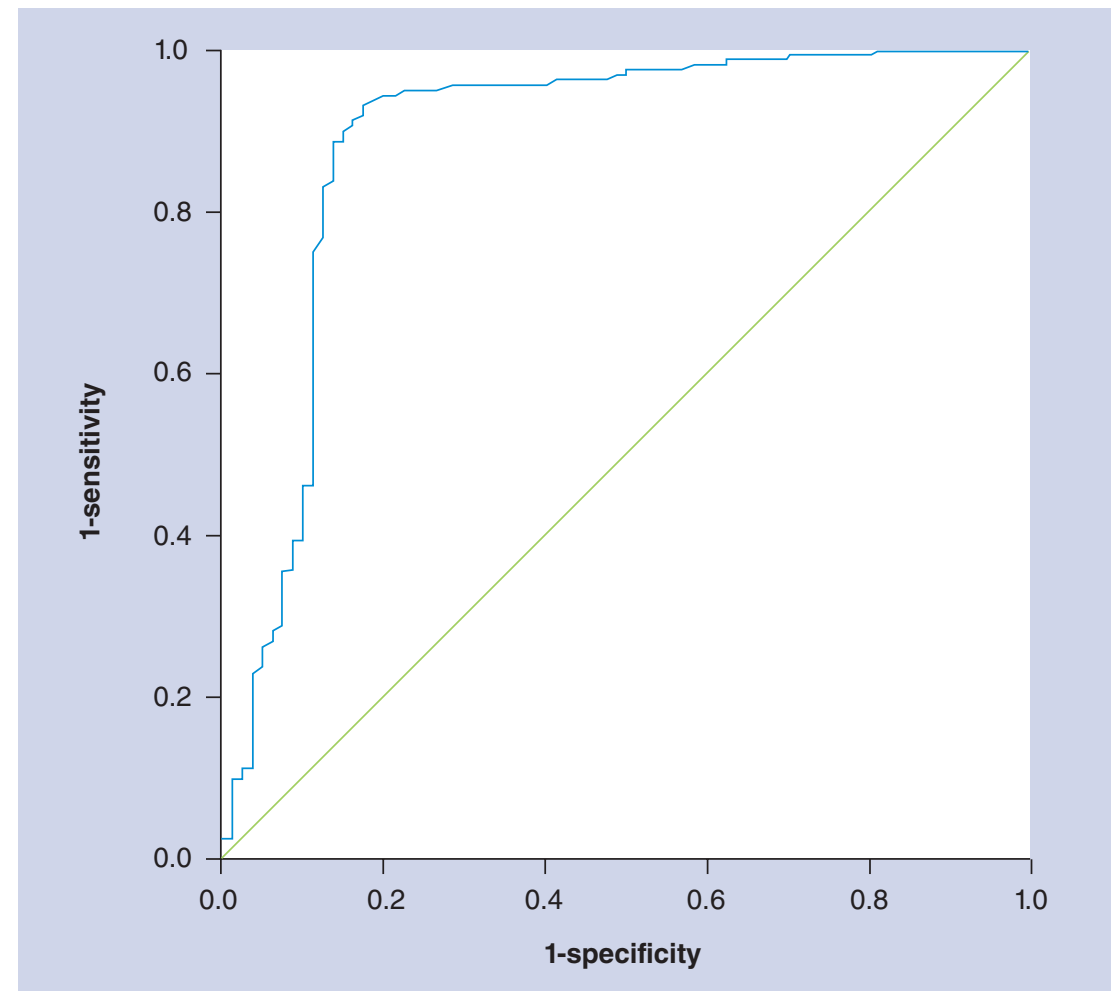

Figure 2. Receiver operator curve for epitope detection in monocytes-TKTL1 score including all 240 individuals (233 confirmed malignancies and seven benign true-negative cases). Diagonal segments are produced by ties.

have been analyzed taking the simultaneous CT findings into consideration. CT data confirmed that five out of $15 \mathrm{FP}$ patients are likely to have small malignancies that were not detected by FDG-PET alone. Thus, the EDIM-TKTL1 score was correct in predicting the presence of small metastases even though FDG-PET showed no increased glucose metabolism. For example, in two patients with breast cancer there was evidence of lesions smaller than $5 \mathrm{~mm}$ on CT, indicating suspicion of lung metastases. Both these lesions did not show any significantly increased FDG

\section{Table 1. Analytical performance by repetitions in intra- and inter-specific epitope} detection in monocyte assays (at three devices).

\begin{tabular}{|lll|}
\hline Analytical performance & Relative amount & Relation \\
\hline Valid replicates below cut-off score 119 & $100 \%$ & $(49 / 49)$ \\
\hline Valid replicates above or equal to 119 & $99 \%$ & $(146 / 148)$ \\
\hline False negative above or equal to 119 & $1 \%$ & $(2 / 148)$ \\
\hline Overall false candidates & $1 \%$ & $(2 / 197)$ \\
\hline SD (intrarun): 11 & CV (intrarun) & $7.7 \%$ \\
\hline SD (interdevice): 7 & CV (interdevice) & $4.9 \%$ \\
\hline SD (total): 13 & CV (total) & $9.1 \%$ \\
\hline CV: Coefficient of variation; SD: Standard deviation. & & \\
\hline
\end{tabular}




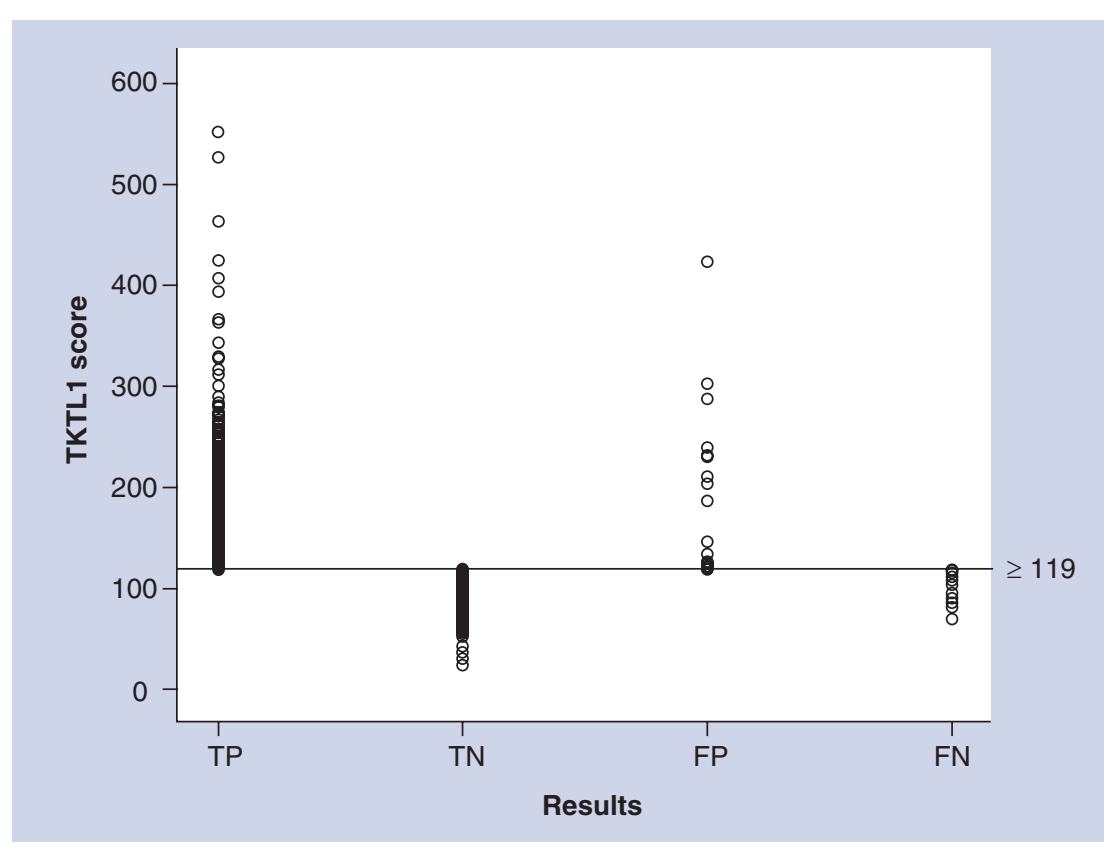

Figure 3. Overall EDIM-TKTL1 score (y-axis) in different patient groups (x-axis).

FN: False-negative cases; FP: False-positive cases; TN: True-negative cases; TP: True-positive cases. positive in the EDIM-TKTL1 test but negative in FDG-PET could be confirmed as having malignancies that were not detected by FDG-PET. Taking these CT results into consideration, the sensitivity and specificity of the EDIM-TKTL1 blood test for detecting malignancies with (or without nonmalignant) diseases was found to be 95\% (regarding 'malignant patients'; i.e., without benign tumors: $95 \%$ ) and $88 \%$ (87\%), whereas PPV and NPV were 95\% (95\%) and 88\% (87\%), respectively (TaвLE 2$)$.

\section{Lung \& breast cancer patients}

A subgroup analysis including the two main tumor entities: lung (NSCLC, $\mathrm{n}=139$; SCLC, $\mathrm{n}=4$ ) and breast $(n=41)$ cancer patients as well as six patients with nonmalignant lung diseases, was also performed to determine diagnostic performance with FDG-PET and FDG-PET/CT as standard, respectively (FIgure 4). There was a significant concordance between the EDIM-TKTL1 score and FDG-PET results, since 173 out of 190 (91\%) patients (with malignant and nonmalignant lung diseases) had identical positive or negative results in the EDIM-TKTL1 blood test and FDG-PET examination, resulting in a sensitivity of $96 \%$ and specificity of $81 \%$. Without nonmalignant lung diseases, there was also a significant concordance between the EDIM-TKTL1 score and FDG-PET results, since 167 out of 184 (91\%) cancer patients had identical positive or negative results in their EDIM-TKTL1 blood test and FDG-PET examination. EDIM-TKTL1 score compared with FDG-PET resulted in a sensitivity and specificity of 96 and $79 \%$, respectively, in cancer patients.

EDIM-TKTL1 results of the whole subgroup analysis (NSCLC and SCLC patients, breast

Table 2. Clinical performance of the epitope detection in monocytes-TKTL1 blood test scores in comparison with PET or PET/computed tomography results.

\begin{tabular}{|c|c|c|c|c|c|c|}
\hline Cohort & Individuals (n) & Concordance & Sensitivity & Specificity & NPV & PPV \\
\hline \multicolumn{7}{|l|}{ PET } \\
\hline All individuals (benign + malignant) & 240 & 89.6 & 93.8 & 81.3 & 86.7 & 90.9 \\
\hline All lung and breast cancer patients & 190 & 91.1 & 95.5 & 81.0 & 88.7 & 92.0 \\
\hline Malignant lung and breast cancer patients & 184 & 90.8 & 95.5 & 78.8 & 87.2 & 92.0 \\
\hline All individuals (benign + malignant) & 240 & 92.5 & 94.5 & 88.0 & 88.0 & 94.5 \\
\hline Malignant patients & 233 & 92.3 & 94.5 & 86.8 & 86.8 & 94.5 \\
\hline All lung and breast cancer patients & 190 & 93.2 & 95.6 & 87.0 & 88.7 & 94.9 \\
\hline Malignant lung and breast cancer patients & 184 & 92.9 & 95.6 & 85.4 & 87.2 & 94.9 \\
\hline
\end{tabular}


cancer patients and nonmalignant lung disease patients) were also compared with FDG-PET/CT data resulting in a sensitivity of $96 \%$ and specificity of $87 \%$. The PPV of the EDIM-TKTL1 blood test was found to be $95 \%$, while the NPV was found to be $89 \%$. EDIM-TKTL1 results of the subgroup analysis without nonmalignant lung diseases were also compared with FDG-PET/CT resulting in a sensitivity of $96 \%$ and specificity of $85 \%$. The PPV of the EDIM-TKTL1 blood test was found to be $95 \%$, while the NPV was found to be $87 \%$. All results of full analysis and subgroup analysis regarding sensitivity, specificity, PPV and NPV are summarized in Table 2.

\section{Correlation of EDIM-TKTL1 score with primary tumor stage of NSCLC patients determined by FDG-PET/CT}

A subgroup analysis of 34 patients with histologically documented NSCLC (mean age $62 \pm 9$ years; 11 men and 23 women) showed a significant correlation $(\mathrm{p}=0.003)$ between the primary tumor stage determined by FDG-PET/CT and the EDIM-TKTL1 score: with increasing tumor stage the EDIM-TKTL1 score also increased (Figure 5) [34]. However, there was no correlation $(\mathrm{p}=0.613)$ between the SUV $_{\max }$ and TKTL1 scores nor between T-stage and $\mathrm{SUV}_{\max }$. Subgroup analysis regarding primary tumor stage of other tumor entities was not performed because data were yet not available.

\section{Discussion}

The metabolic shift from the highly efficient oxygen- and mitochondria-dependent ATP generation to the inefficient anaerobic glucose fermentation to lactate is compensated by an increased glucose uptake and can be visualized by FDG uptake. Although this metabolic switch in cancer cells leads to a glucose dependency, this switch altogether confers a strong selective growth advantage to cancer cells, since this allows oxygen-independent energy release, as well as a suppression of apoptosis and radical induction $[24,35]$. The metabolic shift can be detected by biomarkers specific to this metabolic shift or by detection of enhanced glucose uptake. Since TKTL1 is a biomarker for the glucose fermentation even in the presence of oxygen (aerobic glycolysis/Warburg effect) in cancer cells, the presence of TKTL1 in the cytoplasm of monocytes/macrophages has been evaluated as a blood marker for detecting upregulated glucose metabolism in malignancies.

EDIM technology is a method for detecting intracellular biomarkers in monocytes

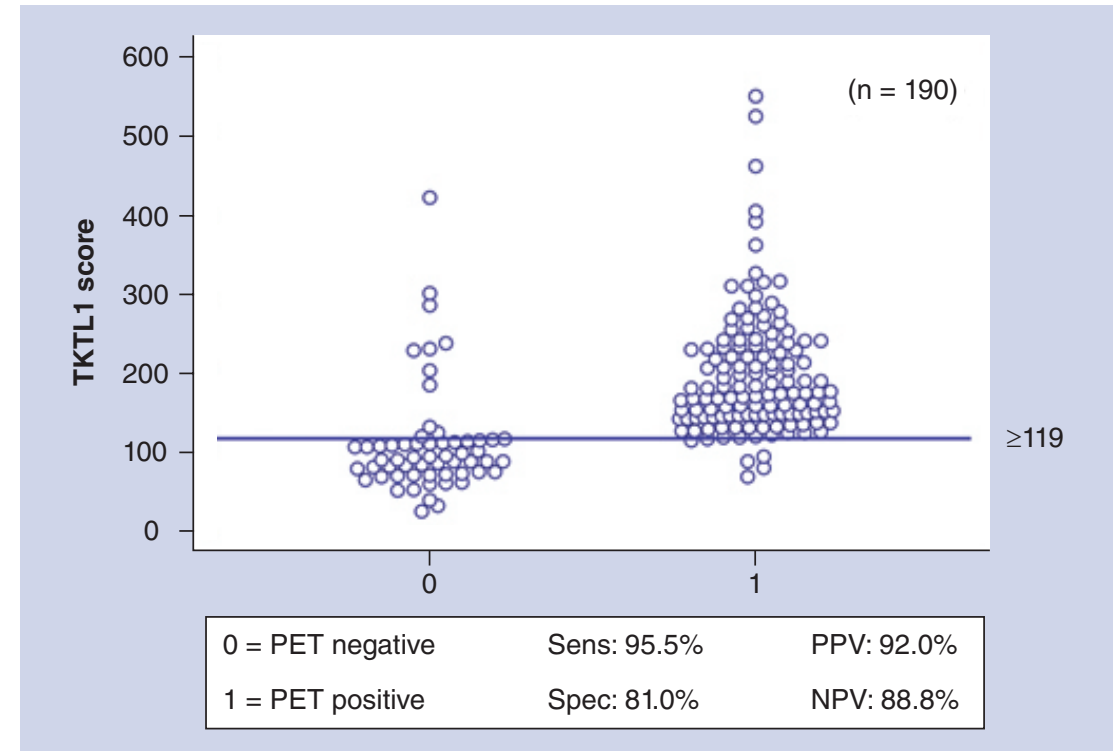

Figure 4. Subgroup analysis including two main tumor entities: non-smallcell lung cancer ( $n=139$; small-cell lung cancer, $n=4)$ and breast cancer patients $(n=41)$ as well as six benign lung diseases shows EDIM-TKTL1 score (Y axis) in PET-negative (0) and in PET-positive (1), respectively. NPV: Negative predictive value; PPV: Positive predictive value; Sens: Sensitivity; Spec: Specificity.

harboring the cellular surface proteins CD14 and CD16 [29-31]. By screening monocytes for a simultaneous expression of $\mathrm{CD}_{14}{ }^{+}$and $\mathrm{CD} 16^{+}$ activated macrophages can be detected with a proinflammatory subtype harboring features of tissue macrophages.

Monocytes and macrophages are involved in nonspecific (innate immunity) as well as specific (adaptive immunity) defensive mechanisms. Their role is to phagocytize cellular debris and pathogens either as sessile or mobile cells and to stimulate immune cells to respond to the pathogen. Macrophages invade solid tissue by ameboid movement through the endothelium of blood vessels. After phagocytosis of the cellular debris, macrophages start digestion of cellular components while returning to the blood stream [30,36]. Thus, activated macrophages harbor tumor-derived protein epitopes that can be detected by antibodies. Therefore, EDIM-technology exploits a complex biological process that obtains access to intracellular proteins in solid tissue in a highly specific and noninvasive manner. Whereas the presence of a biomarker in serum is determined by characteristics as the degree of transition of the biomarker from tumor to serum, by its solubility in water or blood and by the dilution of the blood volume, the presence of a biomarker in macrophages is independent of this, but dependent on the rate of phagocytosis as a consequence of 


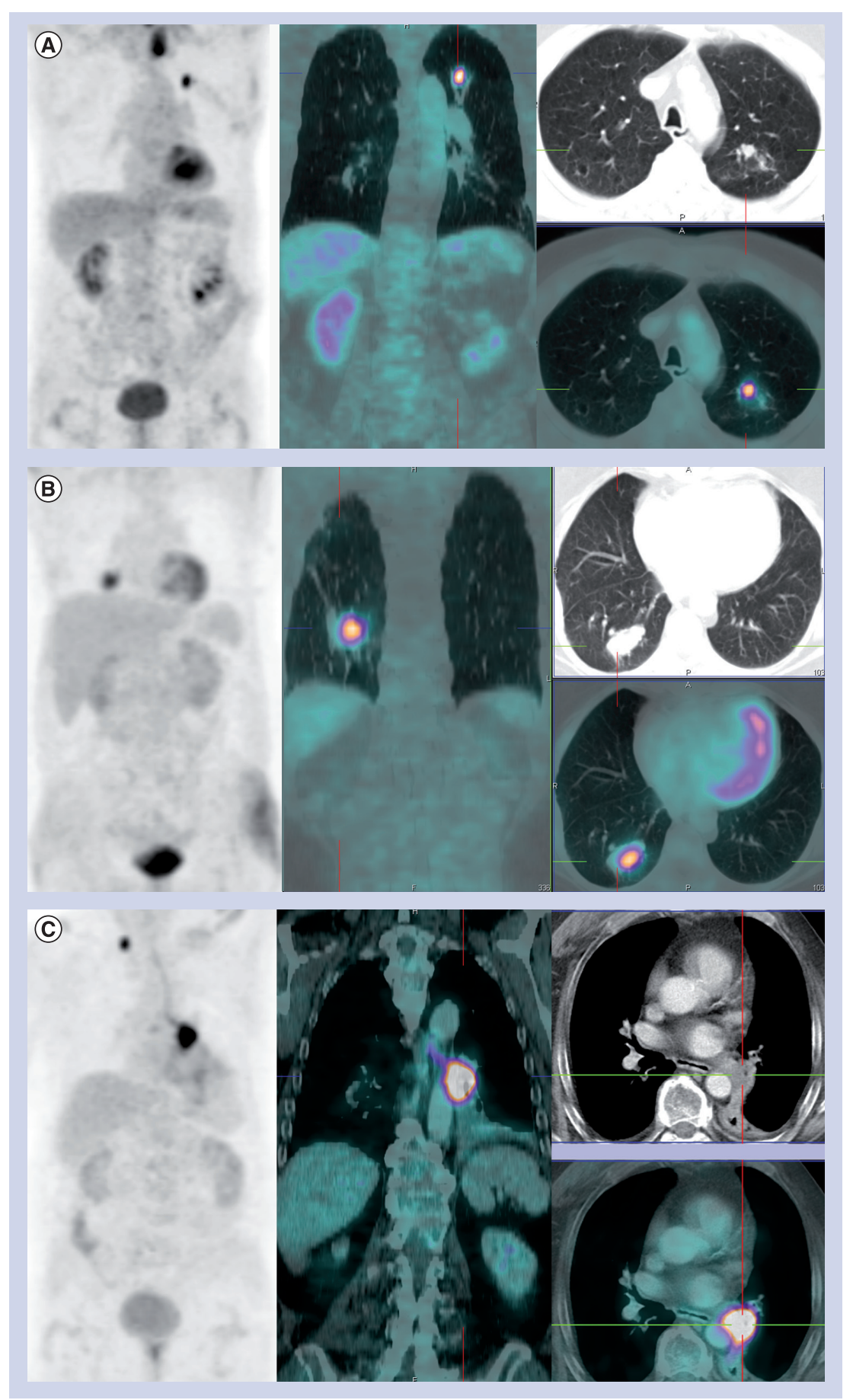

Figure 5. Epitope detection in monocytes-TKTL1 score increases with primary tumor stage as measured by ${ }^{18} \mathrm{~F}-\mathrm{FDG}-\mathrm{PET} / \mathrm{computed}$ tomography in non-small-cell lung cancer patients. (A) Metabolic tumor stage pT1 (standardized uptake value [SUV] 9.3) pNO pM0; TKTL1 score 172. (B) Metabolic tumor stage pT2 (SUV 11.2) pNO CM0; TKTL1 score 232. (C) Metabolic tumor stage pT4 (SUV 24.8) pN3 cM0; TKTL1 score 526.

a specific immune reaction. Therefore, detection of tumor cell-derived biomarkers phagocytized by macrophages also allows the use of biomarkers for the detection and characterization of malignancies. By contrast, tumor markers require a tumor-specific expression, since only the level of tumor marker in serum is detected without cellular context information. Furthermore, adverse to serum biomarkers, the concentration of the biomarker in blood macrophages is not reduced as a consequence of dilution of the blood volume, since the biomarker stays in the cytoplasm of the macrophage even when the macrophage reaches the blood compartment. Therefore, these intrinsic differences between serum- and macrophagebased detection of biomarkers/tumor markers in blood/serum can have a strong impact on specificity and sensitivity of such tests.

Using the EDIM-TKTL1 blood test to detect upregulation of glucose metabolism in a diverse spectrum of malignancies and confirmed nonmalignant alterations, a good concordance to FDG-PET (90\%) has been identified. The EDIM-TKTL1 test was found to have a sensitivity and specificity of 94 and $81 \%$, respectively. A subgroup analysis of the main patient groups with NSCLC and breast cancer including six patients with nonmalignant lung diseases confirmed these results with a sensitivity and specificity of of 96 and $81 \%$, respectively. Taking CT results into consideration (by detection of very small lung and osteoblastic bone lesions), the overall sensitivity and specificity was even higher. It is possible that the reason for the failure of FDG-PET to pick up these small lesions could be related to the small size of the lesions.

Kayser et al. analyzed the NSCLCs of 201 patients for TKTL1 expression by immunohistochemistry [26]. The TKTL1 overexpression correlated with tumor type and histological grading and was significantly associated with poor patient survival. Furthermore, TKTL1 overexpression identified patients with poor clinical outcome among lymph node-negative patients. Diaz-Morelli et al. examined tumor tissues from 63 patients with colorectal cancer. TKTL1 expression increased with tumor staging and high TKTL1 expression correlated with lymph node involvement [28]. In line with those findings, our subgroup analysis of patients with NSCLC showed a significant concordance between the primary tumor size determined by FDG-PET/CT and EDIM-TKTL1 score (Figure 5) [34]. In addition, our results show that even in the T1 stage of NSCLC, TKTL1 was elevated. Langbein et al. observed similar results in the T1 stage of renal cell carcinomas. The subgroup of T1-stage renal cell carcinomas showing strong TKTL1 protein upregulation turned out to be lethal tumors [22]. 
It has to be kept in mind that the EDIM-TKTL1 blood test is based on immune response, so any major impact on the immune system could affect the test results. Patients with a disabled immune system (autoimmune system disorders) may show false results, therefore monitoring of the immune system function (immune status) is recommendable. Also, patients with mitochondrial diseases may show altered test results, as their energy generation depends on glucose fermentation. This may lead to higher TKTL1 levels. Moreover, drugs or therapies that have an inhibiting or stimulating effect on the immune system may affect the test results. For example, immune suppressive therapies as well as radio- and chemo-therapy may alter test results. Further detailed evaluation is needed to evaluate the particular conditions influencing the immune system with regard to the performance of the EDIM-TKTL1 blood test.

\section{Conclusion}

The EDIM-TKTL1 blood test revealed good concordance with FDG-PET/CT results in patients with malignancies, demonstrating its ability to detect upregulation of glucose metabolism in primary tumors or metastases, which is correlated with invasive growth/metastasis and resistance to chemotherapy, radiotherapy and targeted therapies. This could lead to new diagnostic approaches for cancer therapy.

\section{Future perspective}

The reproducible detection of the biomarker TKTL1 in macrophages using the EDIM technology led to results demonstrating that the EDIM-TKTL1 blood test could be used as a new and valid diagnostic test for the detection of increased glucose metabolism in malignant primary tumors and metastases. The good concordance between positive results of the EDIM-TKTL1 blood test and positive FDG-PET and FDG-PET/CT results may allow reliable preselection of cancer patients eligible for FDG-PET/CT imaging before, during and after treatment. Choosing the right time point for FDG-PET/CT often represents a problem, especially in aftercare, when no biomarkers for the detection of recurrences are available. While the EDIM-TKTL1 blood test includes no information on the localization of tumors/metastases, FDG-PET/CT gives detailed information about the localization and distribution of malignancies in the human body. Therefore, the EDIM-TKTL1 blood test and FDG-PET/CT are complementary and synergistic diagnostic approaches to optimize cancer diagnosis and cancer treatment. Since TKTL1 gene activation and upregulation of glucose metabolism in cancer cells is correlated with invasive growth/metastasis and resistance to chemotherapy, radiotherapy and targeted therapy [21], the EDIM-TKTL1 blood test can also be used to detect pretreatment resistance and early detection of in statu nascendi development of resistance to cancer therapies $[23,24,37,38]$.

Financial \& competing interests disclosure
O Feyen, JF Coy and R Schierl are employees of
TAVARLIN AG, Darmstadt, Germany. The authors
have no other relevant affiliations or financial involve-
ment with any organization or entity with a financial
interest in or financial conflict with the subject matter
or materials discussed in the manuscript apart from
those disclosed.
Writing assistance was utilized in the production of
this manuscript. The authors thank E Stetzer,
QuintesScience, Darmstadt, for helpful discussions and
support in drafting and revising this manuscript, which
was funded by TAVARLINAG. The authors also thank
H Hofmann and U Giese, both from TAVARLINAG
Darmstadt, for support in revising this manuscript.

Financial \& competing interests disclosure

$O$ Feyen, JF Coy and $R$ Schierl are employees of TAVARLIN AG, Darmstadt, Germany. The authors have no other relevant affiliations or financial involvement with any organization or entity with a financial interest in or financial conflict with the subject matter or materials discussed in the manuscript apart from those disclosed.

production of QuintesScience, Darmstadt, for help unl discussions and support in drafting and revising this manuscript, which was funded by TAVARLINAG. The authors also thank Darmstadt, for support in revising this manuscript.

\section{Executive summary}

- The new epitope detection in monocytes (EDIM)-TKTL1 blood test is based on EDIM technology, which allows for the detection of circulating activated macrophages harboring TKTL1 via flow cytometry.

- TKTL1 is indicative of glucose fermentation even in the presence of oxygen (aerobic glycolysis/Warburg effect) in cancer cells and can serve as a biomarker for upregulated glucose metabolism.

- The best cut-off score for EDIM-TKTL1 was determined to be 119; scores below 119 are defined as TKTL1 'negative', and those equal to and above 119 are defined as TKTL1 'positive'.

- In blood donors, 94.3\% of blood samples had a negative EDIM-TKTL1 score of less than 119.

- In 240 patients with confirmed or suspected malignancies, EDIM-TKTL1 scores and FDG-PET results (as standard for upregulated glucose metabolism) showed a high concordance of $90 \%$.

- Inclusion of the corresponding computed tomography (CT) data resulted in enhanced values in favor of the EDIM-TKTL1 blood test.

- Subgroup analysis of non-small-cell lung cancer patients showed a significant correlation between the EDIM-TKTL1 score and the primary tumor size determined by FDG-PET/CT.

- Thus, the EDIM-TKTL1 blood test reveals good concordance with FDG-PET/CT results in patients with malignancies. 


\section{Ethical conduct of research \\ The authors state that they have obtained appropriate institutional review board approval or have followed the principles outlined in the Declaration of Helsinki}

for all human or animal experimental investigations. In addition, for investigations involving human subjects, informed consent has been obtained from the participants involved.

\section{References}

Papers of special note have been highlighted as:

- of interest

- of considerable interest

1. Baum RP, Prasad V. Monitoring treatment. In: Clinical Nuclear Medicine. Cook GJR, Maisey MN, Britton KE, Chengazi V (Eds). Hodder Arnold, London, UK, 57-78 (2006).

2. Warburg $\mathrm{OH}$, Posener K, Negelein E. On the metabolism of the cancer cell. Biochemistry 152, 309-344 (1924).

3. Weber G. Enzymology of cancer cells. N. Engl. J. Med. 296, 541-551 (1977).

4. Flier JS, Mueckler MM, Usher P, Lodish HF. Elevated levels of glucose transport and transporter messenger RNA are induced by ras or src oncogenes. Science 235(4795), 1492-1495 (1987).

5. Kelloff GJ, Hoffman JM, Johnson B et al. Progress and promise of FDG-PET imaging for cancer patient management and oncologic drug development. Clin. Cancer Res. 11(8), 2785-2808 (2005).

6. von Schulthess GK, Steinert HC, Hany TF. Integrated PET/CT: current applications and future directions. Radiology 238 (2), 405-422 (2006).

7. Baum RP, Swietaszczyk C, Prasad V. FDG-PET/CT in lung cancer: an update. Front. Radiat. Ther. Oncol. 42, 15-45 (2010).

- Illustrates that the combination of PET and computed tomography is extremely useful in tumor imaging and combining with fluoro-2-deoxy-D-glucose makes it an important tool in tumor staging and therapy-monitoring applications.

8. Hellwig D, Baum RP, Kirsch C. FDG-PET, $\mathrm{PET} / \mathrm{CT}$ and conventional nuclear medicine procedures in the evaluation of lung cancer: a systematic review. Nuklearmedizin 48(2), 59-69; quiz N8-N9 (2009).

9. Lee JH, Rosen EL, Mankoff DA. The role of radiotracer imaging in the diagnosis and management of patients with breast cancer: part 1 - overview, detection, and staging. J. Nucl. Med. 50 (4), 569-581 (2009).

10. Lee JH, Rosen EL, Mankoff DA. The role of radiotracer imaging in the diagnosis and management of patients with breast cancer: part 2 - response to therapy, other indications, and future directions. J. Nucl. Med. 50(5), 738-748 (2009).
11. Weiler-Sagie M, Bushelev O, Epelbaum R et al. (18)F-FDG avidity in lymphoma readdressed: a study of 766 patients. J. Nucl. Med. 51(1), 25-30 (2010).

12. Fletcher JW, Djulbegovic B, Soares HP et al. Recommendations on the use of $18 \mathrm{~F}-\mathrm{FDG}$ PET in oncology. J. Nucl. Med. 49(3), 480-508 (2008).

13. Pfister DG, Johnson DH, Azzoli CG et al. American society of clinical oncology treatment of unresectable non-small-cell lung cancer guideline: update 2003. J. Clin. Oncol. 22(2), 330-353 (2004).

14. Palaskas N, Larson SM, Schultz N et al. 18F-FDG PET marks MYC-overexpressing human basal like breast cancers. Cancer Res. 71(15), 5164-5174 (2011).

15. Riganti C, Gazzano E, Polimeni M, Aldieri E, Ghigo D. The pentose phosphate pathway: an anti-oxidant defense and a cross-road in tumor cell fate. Free Radic. Biol. Med. 53(3), 421-436 (2012).

16. Langbein S, Zerilli M, Zur Hausen A et al. Expression of transketolase TKTL1 predicts colon and urothelial cancer patient survival: warburg effect reinterpreted. Br. J. Cancer 94 , 578-585 (2006).

-. Shows the upregulation of TKTL1 in human malignancies and the important correlation with poor patient outcome in colon and urothelial cancers.

17. Kohrenhagen N, Voelker HU, Schmidt M et al. Expression of transketolase-like 1 (TKTL1) and p-Akt correlates with the progression of cervical neoplasia. J. Obstet. Gynaecol. Res. 34, 293-300 (2008).

18. Zerilli M, Amato MC, Martorana A et al. Increased expression of transketolase-like-1 in papillary thyroid carcinomas smaller than $1.5 \mathrm{~cm}$ in diameter is associated with lymph-node metastases. Cancer 113, 936-944 (2008).

19. Krockenberger M, Honig A, Rieger L et al. Transketolase-like 1 expression correlates with subtypes of ovarian cancer and the presence of distant metastases. Int. J. Gynecol. Cancer 17, 101-106 (2007).

20. Schmidt M, Voelker HU, Kapp M, Krockenberger M, Dietl J, Kammerer U. Glycolytic phenotype in breast cancer: activation of Akt, upregulation of GLUT1, TKTL1 and down-regulation of M2PK. J. Cancer Res. Clin. Oncol. 136(2), 219-225 (2010).
21. Schwaab J, Horisberger K, Strobel P et al. Expression of transketolase like gene 1 (TKTL1) predicts disease-free survival in patients with locally advanced rectal cancer receiving neoadjuvant chemoradiotherapy. Biomed. Cancer 11, 363 (2011).

- In patients with rectal cancer that are treated with chemoradiotherapy and targeted therapy, high TKTL1 expression is the only molecular marker that correlates with poor prognosis.

22. Langbein S, Frederiks WM, zur Hausen A et al. Metastasis is promoted by a bioenergetic switch: new targets for progressive renal cell cancer. Int. J. Cancer 122(11), 2422-2428 (2008).

23. Sun W, Liu Y, Glazer CA et al. TKTL1 is activated by promoter hypomethylation and contributes to head and neck squamous cell carcinoma carcinogenesis through increased aerobic glycolysis and HIF1 $\alpha$ stabilization. Clin. Cancer Res. 16(3), 857-866 (2010).

24. Xu X, Zur Hausen A, Coy JF, Löchelt M. Transketolase-like protein 1 (TKTL1) is required for rapid cell growth and full viability of human tumor cells. Int. J. Cancer 124(6), 1330-1337 (2009).

25. Hu LH, Yang JH, Zhang DT et al. The TKTL1 gene influences total transketolase activity and cell proliferation in human colon cancer LoVo cells. Anticancer Drugs 18(4), 427-433 (2007).

26. Kayser G, Sienel W, Kubitz B et al. Poor outcome in primary non-small cell lung cancers is predicted by transketolase TKTL1 expression. Pathology 43(7), 719-724 (2011).

- Shows that TKTL1 overexpression is a new and independent prognostic marker for the survival of patients with non-small-cell lung cancer.

27. Lange CA, Tisch-Rottensteiner J, Böhringer D, Martin G, Schwartzkopff J, Auw-Haedrich C. Enhanced TKTL1 expression in malignant tumors of the ocular adnexa predicts clinical outcome. Ophthalmology 119(9), 1924-1929 (2012).

28. Diaz-Moralli S, Tarrado-Castellarnau M, Alenda C, Castells A, Cascante M. Transketolase-like 1 expression is modulated during colorectal cancer progression and metastasis formation. PLoS ONE 6, e25323 (2011).

29. Herwig R, Pelzer A, Horninger W et al. Measurement of intracellular versus extracellular prostate specific antigen levels in 
peripheral macrophages: a new approach to non-invasive diagnosis of prostate cancer.

Clin. Prostate Cancer 3(3), 184-188 (2004).

30. Leers MP, Nap M, Herwig R, Delaere K, Nauwelaers F. Circulating PSA-containing macrophages as a possible target for the detection of prostate cancer. Am. J. Clin. Pathol. 129, 649-656 (2008).

- Shows that epitopes of tumor markers such as prostate-specific antigen can be detected in circulating macrophages and this method could be used to differentiate between malignant and benign disease.

31. Japink D, Leers MP, Sosef MN, Nap M. CEA in activated macrophages. New diagnostic possibilities for tumor markers in early colorectal cancer. Anticancer Res. 29, 3245-3251 (2009).
32. Boellaard R, O'Doherty MJ, Weber WA et al. FDG PET and PET/CT: EANM procedure guidelines for tumour PET imaging: version 1.0. Eur. J. Nucl. Med. Mol. Imaging 37, 181-200 (2010).

33. Prasad V, Baum RP. Biodistribution of the Ga-68 labeled somatostatin analogue DOTA-NOC in patients with neuroendocrine tumors: characterization of uptake in normal organs and tumor lesions. Q. J. Nucl. Med. Mol. Imaging 54, 61-67 (2010).

34. Prasad V, Mehlhorn U, Feyen O, Coy J, Baum RP. Transketolase-like 1 (TKTL1), a new intracellular marker for staging of non-small cell lung cancer (NSCLC): correlation with metabolic tumor volume on F-18 FDG PET/CT. J. Nucl. Med. 51(Suppl. 2), 1253 (2010).
35. Hartmannsberger D, Mack B, Eggert C et al. Transketolase-like protein 1 confers resistance to serum withdrawal in vitro. Cancer Lett. 300, 20-29 (2011).

36. Parihar A, Eubank TD, Doseff AL. Monocytes and macrophages regulate immunity through dynamic networks of survival and cell death. J. Innate Immun. 2, 204-215 (2010).

37. Tennant DA, Durán RV, Gottlieb E. Targeting metabolic transformation for cancer therapy. Nat. Rev. Cancer 10, 267-277 (2010).

38. Furuta E, Okuda H, Kobayashi A, Watabe K. Metabolic genes in cancer: their roles in tumor progression and clinical implications. Biochim. Biophys. Acta 1805 , 141-152 (2010). 